\title{
Exploring molecular mysteries of the adrenal gland: Start with the basics
}

\author{
Victor A. Ferraris, MD, PhD
}

\footnotetext{
From the Division of Cardiothoracic Surgery, Department of Surgery, University of Kentucky A301 Kentucky Clinic, Lexington, Ky.

Disclosures: Author has nothing to disclose with regard to commercial support.

Received for publication March 24, 2018; accepted for publication March 28, 2018; available ahead of print April $21,2018$.

Address for reprints: Victor A. Ferraris, MD, PhD, Division of Cardiothoracic Surgery, Department of Surgery, University of Kentucky, A301 Kentucky Clinic, 740 S Limestone, Lexington, KY 40536-0284 (E-mail: ferraris@uky.edu).

J Thorac Cardiovasc Surg 2018;156:707-8

$0022-5223 / \$ 36.00$

Copyright (c) 2018 by The American Association for Thoracic Surgery

https://doi.org/10.1016/j.jtcvs.2018.03.131
}

The functional anatomy and physiology of the adrenal gland were fresh in the mind of the average cardiothoracic surgeon at some time during medical school training but were relegated to a slightly mysterious memory after that. The article by Mei and coauthors ${ }^{1}$ in the current issue of the Journal challenges that memory. In this issue, Mei and coauthors ${ }^{1}$ explore the adrenal gland molecular components of the inflammatory response to cardiopulmonary bypass (CPB). Extension of advances in molecular biology and molecular mechanisms to the adrenal gland offers new avenues that can affect outcomes after CPB.

Evidence supports the notion that there is relative adrenocortical insufficiency after CPB. This deficit is worsened by some anesthetic agents and is likely to cause more prominent findings of adrenocortical deficit in neonatal CPB. ${ }^{2-4}$ The impacts of the relative adrenal insufficiency and results of exogenously administered adrenal hormones during CPB are uncertain. A Cochrane Review suggests that post-CPB systemic inflammatory response syndrome is resistant to addition of corticosteroids during and after the operation. ${ }^{5}$ A subsequent meta-analysis found that steroid prophylaxis in patients undergoing coronary artery bypass grafting significantly reduced complications such as atrial fibrillation and prolonged hospital stay but slightly increased the duration of ventilation. ${ }^{6}$ Similarly, the infectious risks of adrenocortical insufficiency and of administering exogenous steroids in the perioperative setting are uncertain. 6,7

How should we respond to these adrenal function uncertainties during CPB? The short answer is to start with the basics. The basics involve the molecular substrates of $\mathrm{CPB}$ and the inflammatory response. The article by Mei and coauthors ${ }^{1}$ in the current issue of the Journal begins the process of investigating the molecular underpinnings of the adrenal injury and of the inflammatory response associated with CPB. Mei and coauthors ${ }^{1}$ studied adrenocortical cellular and molecular pathways in normal control dogs and in dogs exposed to CPB. They found that CPB induces local from CPB. new information.

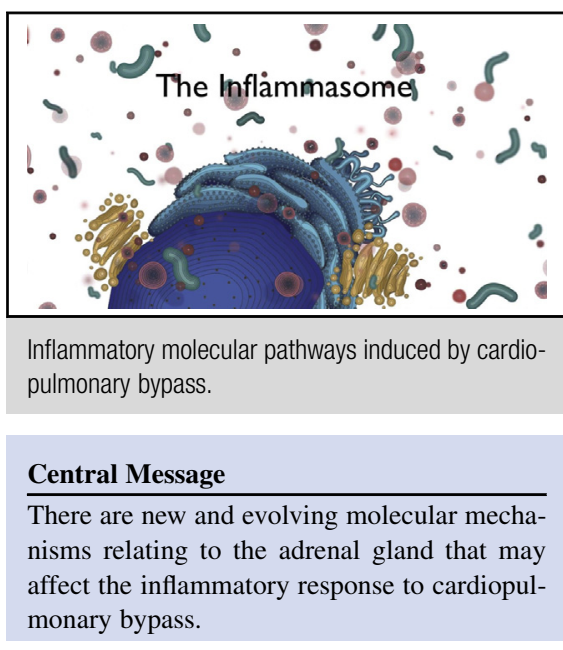

See Article page 696.

inflammatory changes in the adrenal cortex including changes in architecture and in inflammatory molecular pathways. They introduce terms that might seem like reading ancient Greek to the average cardiothoracic surgeon. Terms such as inflammasome and nucleotide-binding oligomerization domain-like receptor containing pyrin domain $3^{\prime}$ [NLRP3] are not used in everyday conversation by most cardiothoracic surgeons. Nonetheless, these terms encompass the beginnings of the understanding of the adrenal gland molecular response to the inflammatory stimulus

It is well known that CPB induces an inflammatory response and injury in sensitive organs such as the heart, lungs, and kidneys. ${ }^{8-10}$ In their animal model, Mei and coauthors ${ }^{1}$ found that $\mathrm{CPB}$ induces significant local adrenal cortical inflammation resulting in cytological alterations. These adrenal inflammatory changes occurred along with a significant systemic inflammatory response. Mei and coauthors $^{1}$ found activation of one inflammasome pathway (NLRP3) that may be a target for intervention in subsequent studies. This NLRP3 inflammasome is a multiprotein oligomer that promotes the maturation and secretion of proinflammatory cytokines, especially interleukins $1 \beta$ and $18 .{ }^{11}$ These oligomers are expressed in myeloid cells, but their finding in adrenal cells, although not surprising, is relatively

Mei and coauthors ${ }^{1}$ suggest that NLRP3 plays a prominent role in the adrenocortical component of CPB- 
induced inflammation. They outlined the upregulation of NLRP3 and identified several proinflammatory actions, including interleukin $1 \beta$ expression. To implicate NLRP3 as a prominent mediator of the post-CPB inflammatory response, it would be necessary to identify the priming signal for NLRP3 expression and the targets after expression. A complete definition of the role of NLRP3 was beyond the study scope of Mei and coauthors, ${ }^{1}$ but the possibilities are intriguing and should be explored. Ultimately, these studies may lead to new molecular targets to limit the harmful components of the CPB-induced inflammatory response. Studies directed at the $\mathrm{CPB}$-induced cellular triggers for NLRP3 upregulation, and the NLRP3-induced cellular actions, may identify new targets to limit harmful proinflammatory responses. The article by Mei and coauthors ${ }^{1}$ provides a glimpse into future experiments and is a beginning step in unravelling the molecular mechanisms involving the adrenal gland during CPB.

There are some questions about the study groups that Mei and coauthors ${ }^{1}$ used. Clinically, many surgeons attempt to lessen the CPB-induced inflammatory response with empirically based steroid therapy during the perioperative period. I am surprised that Mei and coauthors ${ }^{1}$ did not include a third group of dogs in their experiments. Specifically, a group of dogs that received high-dose steroids during CPB would have been an informative control group. There are multiple strategies to attenuate this response other than the administration of corticosteroids, including aprotinin and anticytokine monoclonal antibodies, as well as various modifications of the bypass circuit. CPB induces inflammation and subsequent organ dysfunction. It is very difficult to look only at the adrenal cortex in isolation as a trigger of the inflammatory response and of CPB-induced organ dysfunction without also considering multiple other organ-system effects of CPB. The interest of the readers revolves around clinical pathways and CPB outcomes. Mei and coauthors ${ }^{1}$ identified specific post-CPB abnormalities associated with a single adrenocortical pathway activation. The clinical implications of their initial observations need to be expanded and defined with precision. One hopes that human trials, and ultimately clinical benefits, will follow this preliminary work.

\section{References}

1. Mei B, Yang S, Yue Y, Hou J, Wang K, Chen G, et al. Acute adrenal cortex injury during cardiopulmonary bypass in a canine model. J Thorac Cardiovasc Surg. 2018;156:696-706.

2. Iribarren JL, Jiménez JJ, Hernández D, Lorenzo L, Brouard M, Milena A, et al. Relative adrenal insufficiency and hemodynamic status in cardiopulmonary bypass surgery patients. A prospective cohort study. J Cardiothorac Surg. 2010;5:26

3. Das D, Sen C, Goswami A. Effect of vitamin C on adrenal suppression by etomidate induction in patients undergoing cardiac surgery: a randomized controlled trial. Ann Card Anaesth. 2016;19:410-7.

4. Crawford JH, Hull MS, Borasino S, Steenwyk BL, Hock KM, Wall K, et al. Adrenal insufficiency in neonates after cardiac surgery with cardiopulmonary bypass. Paediatr Anaesth. 2017;27:77-84.

5. Dieleman JM, van Paassen J, van Dijk D, Arbous MS, Kalkman CJ, Vandenbroucke JP, et al. Prophylactic corticosteroids for cardiopulmonary bypass in adults. Cochrane Database Syst Rev 2011;5:CD005566.

6. Ali-Hassan-Sayegh S, Mirhosseini SJ, Haddad F, Karimi-Bondarabadi AA, Shahidzadeh A, Weymann A, et al. Protective effects of corticosteroids in coronary artery bypass graft surgery alone or combined with valvular surgery: an updated and comprehensive meta-analysis and systematic review. Interact Cardiovascular Thorac Surg. 2015;20:825-36.

7. Robertson-Malt S, Afrane B, El Barbary M. Prophylactic steroids for pediatric open heart surgery. Cochrane Database Syst Rev 2007;4:CD005550.

8. Whitlock RP, Devereaux PJ, Teoh KH, Lamy A, Vincent J, Pogue J, et al. Methylprednisolone in patients undergoing cardiopulmonary bypass (SIRS): a randomised, double-blind, placebo-controlled trial. Lancet. 2015;386: 1243-53.

9. Belley-Côté EP, Parikh CR, Shortt CR, Coca SG, Garg AX, Eikelboom JW, et al. Association of cardiac biomarkers with acute kidney injury after cardiac surgery: a multicenter cohort study. J Thorac Cardiovasc Surg. 2016;152: 245-51.e4.

10. Adamik B, Kübler A, Gozdzik A, Gozdzik W. Prolonged cardiopulmonary bypass is a risk factor for intestinal ischaemic damage and endotoxaemia. Heart Lung Circ. 2017;26:717-23.

11. Martinon F, Burns K, Tschopp J. The inflammasome: a molecular platform triggering activation of inflammatory caspases and processing of proIL- $\beta$. Mol Cell. 2002;10:417-26. 